Document downloaded from:

http://hdl.handle.net/10251/49849

This paper must be cited as:

Bernal García, A.; Abarca Giménez, A.; Barrachina Celda, TM.; Miró Herrero, R. (2014). Methodology to resolve the transport equation with the discrete ordinates code TORT into the IPEN/MB-01 reactor. International Journal of Computer Mathematics. 91(1):113-123. doi:10.1080/00207160.2013.799668.

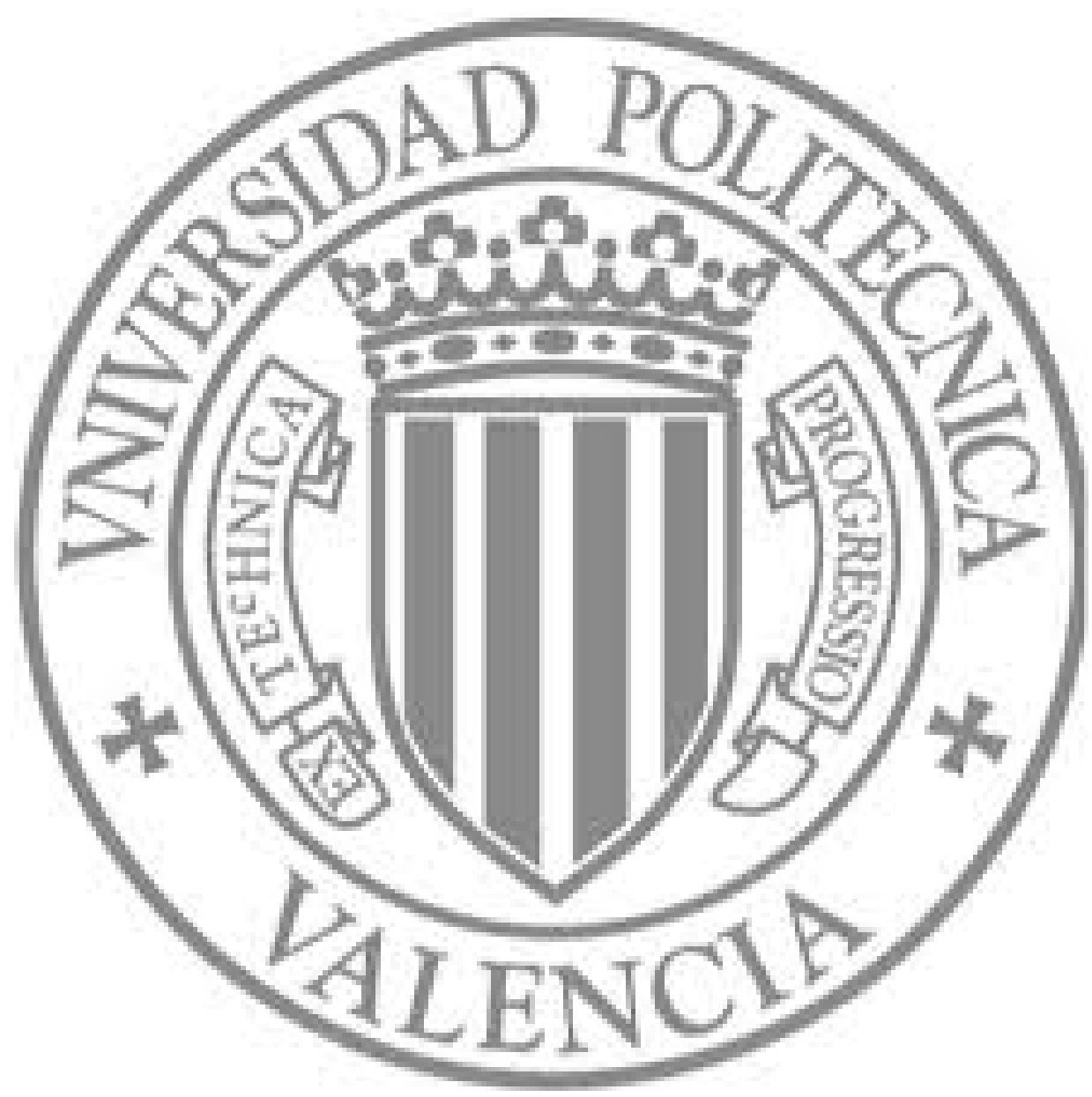

The final publication is available at

http://dx.doi.org/10.1080/00207160.2013.799668

Copyright Taylor and Francis 


\title{
Methodology to resolve the transport equation with the discrete ordinates code TORT into IPEN/MB-01 reactor.
}

\author{
A. Bernal ${ }^{1}$, A. Abarca ${ }^{1}$, T. Barrachina ${ }^{1}$ and R. Miró ${ }^{1}$. \\ ${ }^{1}$ Institute for Industrial, Radiophysical and Environmental Safety (ISIRYM), Universitat \\ Politècnica de València, Valencia, Spain.
}

abernal@iqn.upv.es, aabarca@iqn.upv.es, tbarrachina@iqn.upv.es, rmiro@iqn.upv.es. 


\title{
Methodology to resolve the transport equation with the discrete ordinates code TORT into IPEN/MB-01 reactor.
}

\begin{abstract}
Resolution of the steady state Neutron Transport Equation in a nuclear pool reactor is usually achieved by means of two different numerical methods: Monte Carlo (stochastic) and Discrete Ordinates (deterministic). The Discrete Ordinates Method solves the Neutron Transport Equation for a set of selected directions, obtaining a set of directional equations and solutions for each equation which are the angular flux. In order to deal with the energy dependence, an energy multigroup approximation is commonly performed, obtaining a set of equations depending on the number of energy groups. In addition, spatial discretization is also required and the problem is solved by sweeping the geometry mesh. However, special cross sections are required due to the energy and directional discretization, thus a methodology based on NJOY99 code capabilities has been used. Finally, in order to demonstrate the capability of this method, the 3D discrete ordinates code TORT has been applied to resolve the IPEN/MB-01 reactor.
\end{abstract}

Keywords: discrete ordinates, transport equation, TORT.

\section{Introduction}

\section{Nuclear reactors}

The neutron distribution inside actual reactor cores is computed by codes based on the Neutron Diffusion Theory. However, the Diffusion Theory is a simplification of the Transport Theory which requires special cross sections treatment to be applied for advanced reactors as opposed to Transport Theory. Moreover, Transport Theory offers more detailed results, but it requires more and sometimes computational resources.

The resolution of the steady state Neutron Transport Equation, using the Transport Theory, is usually achieved by means of two different numerical methods [1]: Monte Carlo (stochastic) and Discrete Ordinates (deterministic). 


\section{Monte Carlo and Deterministic methods}

Monte Carlo method solves the Transport Equation by simulating the different events that can occur in the reactor, which are the nuclear interactions and reactions. In this method, these events are simulated in a sequential form by taking into account the probabilities of occurrence and using random numbers, thus, it is a stochastic method [2]. Therefore, the resolution of the problem is equivalent to a sampling of the different events.

The major capability of the Monte Carlo method is the resolution of complex problems in terms of energy and geometry. It can solve continuous energy problems and different types of geometry due to the fact that it does not deal with the integral form of the Transport Equation. Nevertheless, this method implies errors because of the stochastic resolution, than can be reduced by increasing the number of events simulated, thereby, increasing the computational time.

On the other hand, Deterministic methods solve the integro-differential Transport Equation by discretizing the phase space: energy, direction and space. In particular, the Discrete Ordinates method [3] resolves the Transport Equation for a set of selected directions (quadrature sets), obtaining a set of directional equations and solutions for each equation which are the angular flux. The final solution is the weighted sum of all the directional solutions. In order to deal with the energy dependence, an energy multi-group approximation is commonly performed, obtaining a set of equations depending on the number of energy groups. In this case, the final solution will be the sum of the solutions for each group. Finally, space discretization is solved by means of a sweep of the different meshes. Summarizing, the obtained equations are an approximation of the real problem, but the resolution of this approximation is deterministic. 
The major capability of the Discrete Ordinates method is the deterministic resolution of the equation. However, it can be only applied to discrete energies and simple geometries.

\section{Cross sections for deterministic methods}

Cross Sections are the probabilities of interaction of the different kinds of nuclear reactions. They are necessary to compute the Transport Equation (equation coefficients). Moreover, they depend strongly on energy and may depend on direction.

In the Deterministic method with the two discretizations, energy and direction, special cross-sections are needed. On one hand, the production (fission) and total cross sections are needed in terms of group energy cross-sections, but there is not directional dependence due to the isotropic behavior of them. On the other hand, the scattering cross-sections must be specified by energy and by direction, due to the double differential scattering cross-section. Therefore, the Legendre Expansion can be used to define the directional dependence; thus, scattering cross-section must be defined for each energy group and term of the Legendre Expansion.

We have used a methodology to obtain the multi-group nuclear cross-sections data, in the fast and thermal energy range, at the temperature of interest, based on the capabilities of NJOY99 code [4].

\section{Resolution of the transport equation with the discrete ordinates method}

The time dependent neutron transport equation in integro-differential form can be written as:

$$
\begin{gathered}
\frac{1}{v} \frac{\partial \psi(\vec{r}, E, \widehat{\Omega}, t)}{\partial t}+\widehat{\Omega} \nabla \psi(\vec{r}, E, \widehat{\Omega}, t)+\Sigma_{t}(\vec{r}, E) \psi(\vec{r}, E, \widehat{\Omega}, t)=\int_{4 \pi} d \widehat{\Omega}^{\prime} \int_{0}^{\infty} d E^{\prime} \Sigma_{s}\left(E^{\prime}, \widehat{\Omega}^{\prime} \rightarrow\right. \\
E, \widehat{\Omega}) \psi\left(\vec{r}, E^{\prime}, \widehat{\Omega}^{\prime}, t\right)+\frac{\chi(E)}{4 \pi} \int_{0}^{\infty} d E^{\prime} v\left(E^{\prime}\right) \Sigma_{f}\left(\vec{r}, E^{\prime}\right) \int_{4 \pi} d \widehat{\Omega}^{\prime} \psi\left(\vec{r}, E^{\prime}, \widehat{\Omega}^{\prime}, t\right)+ \\
s(\vec{r}, E, \widehat{\Omega}, t)
\end{gathered}
$$


Where $v$ is the neutron velocity, $\psi$ is the unknown flux, $\vec{r}$ is the space vector, $E$ is the energy of the neutron, $\widehat{\Omega}$ is the solid angle of the neutron direction, $t$ is the time, $\Sigma_{t}$ is the total macroscopic cross section, $\Sigma_{s}\left(E^{\prime}, \widehat{\Omega}^{\prime} \rightarrow E, \widehat{\Omega}\right)$ is the double differential scattering macroscopic cross section, $\chi$ is the fission spectrum, while $\Sigma_{f}$ is the fission macroscopic cross section and $s$ is a neutron source.

The terms at the left side of equation 1 represent the losses of neutrons of energy $E$ and direction $\widehat{\Omega}$ : variation in time, leakage and total collision. On the other hand, the terms of the right-hand side of the equation 1 are the sources of neutrons of energy $E$ and direction $\widehat{\Omega}$ : scattering, fission and fixed-source.

The Discrete Ordinates method implies 3 discretizations to deal with the neutron transport equation: energy, direction and space. The energy discretization is dealt with the multigroup approach that replaces the continuum variation of energy by energy bins:

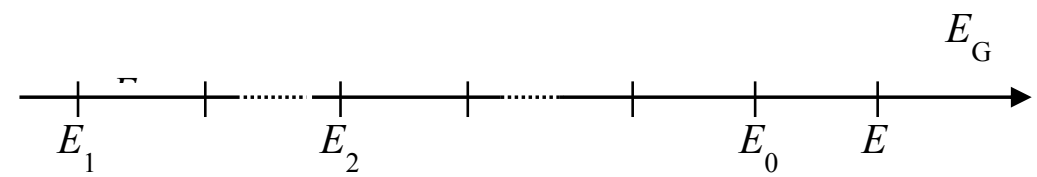

Figure 1. Multigroup approach.

Therefore, the dependent functions of the energy are replaced by $g$ functions that are the integral over their respective energy bins:

$$
\begin{gathered}
\phi_{g}=\int_{E_{g}}^{E_{g-1}} \phi(E) d E \\
\Sigma_{g}=\frac{\int_{E_{g}}^{E_{g-1}} \Sigma(E) \phi(E) d E}{\int_{E_{g}}^{E_{g}-1} \phi(E) d E}=\frac{\int_{E_{g}}^{E_{g-1}} \Sigma(E) \phi(E) d E}{\phi_{g}}
\end{gathered}
$$

Equation 2 represents the group angular flux and equation 3 the multigroup cross sections. It is important to note that the cross section is also energy dependent and the multigroup cross section depend on the flux, which is the solution of the problem, 
but it is unknown. However, they depend on these variables in a continuous range, but the cross sections do not depend on the group flux. There are several methodologies to obtain the multigroup cross sections using estimations of the flux for any energy bin [3].

On the other hand, the directional dependence is treated by choosing several directions and resolving the equation for these selected directions. One equation and solution, which is the angular flux, is obtained for each direction. Nonetheless, the final solution will be the sum of all the directional solutions, that is, the sum of all the angular fluxes [5].

Therefore, the Discrete Ordinates approximation to the transport equation has 7 variables that do not depend in energy, direction and space, because the equation is solved for a certain group of energy, direction and cell of the space. However, there is only one equation and 7 unknowns; if the first node of a discretized mesh (corresponding to a corner cell) of the problem is considered, it has 3 boundary

conditions which are the input boundary fluxes: $\psi_{i-\frac{1}{2}, j, k}, \psi_{i, j-1 / 2, k}, \psi_{i, j, k-1 / 2}$.Thus, 3 more relationships are required to solve this transport equation. To compute this problem, the diamond difference model could be used [3].

Finally, the solution of the transport equation is the average flux in each mesh. On the other hand, boundary fluxes can be obtained by the diamond difference/step relationships [3], input boundary fluxes and average flux. In addition, the output boundary fluxes will represent the input boundary fluxes for the next mesh and all the geometry can be solved by sweeping all the meshes, but the process requires an iteration procedure.

\section{Results}

With the aim of showing the capabilities of this method, the IPEN/MB-01 reactor has 
been simulated under certain conditions with TORT code.

TORT is a computer code which solves the neutron transport equation and reactor eigenvalue problems in steady state conditions in 3D geometries. It calculates flux or fluence, using the Discrete Ordinates method. It was prepared by the Oak Ridge National Laboratory [5].

The IPEN/MB-01 research reactor is a zero power critical facility specially designed for measurement of a wide variety of reactor physics parameters to be used as benchmark experimental data for checking the calculation methodologies and related nuclear data libraries commonly used in the field of reactor physics. It is a pool reactor and consists of a $28 \times 26$ rectangular array of $\mathrm{UO}_{2}$ fuel rods $4.3486 \%$ (weight) enriched, with a stainless steel (SS-304) clad inside a light water tank. The control of the reactor is performed via two control rod banks, diagonally placed. The control banks are composed of $12 \mathrm{Ag}$-In-Cd rods and the safety banks by $12 \mathrm{~B}_{4} \mathrm{C}$ rods [6].

In order to compare the results, another simulation with the MCNP code [2], which is a Monte Carlo code, has been done. Moreover, there are experimental results in the International Handbook of Evaluated Reactor Physics Benchmark Experiments [6] which can be used for comparison, such as the fission rate. TORT calculates fluxes, but they can be transformed in fission rate by multiplying the fluxes by the fission macroscopic cross sections, which are known. In fact, this operation can be introduced into TORT, so the obtained solution will be fission rate.

In the following figures the reactor model is shown: 


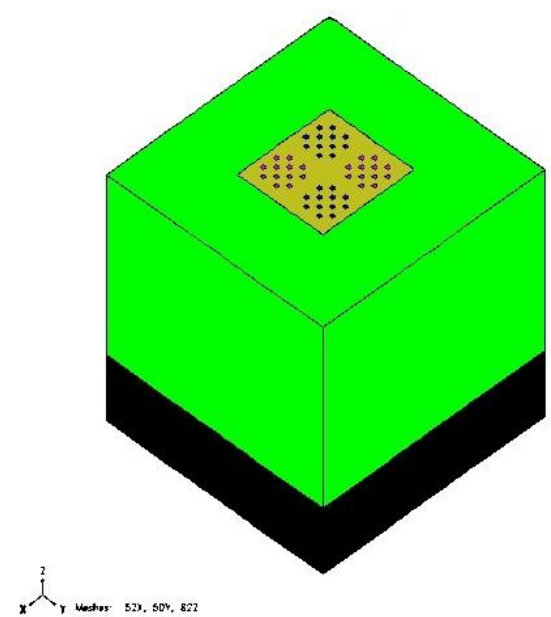

Figure 2.3D view of the reactor model

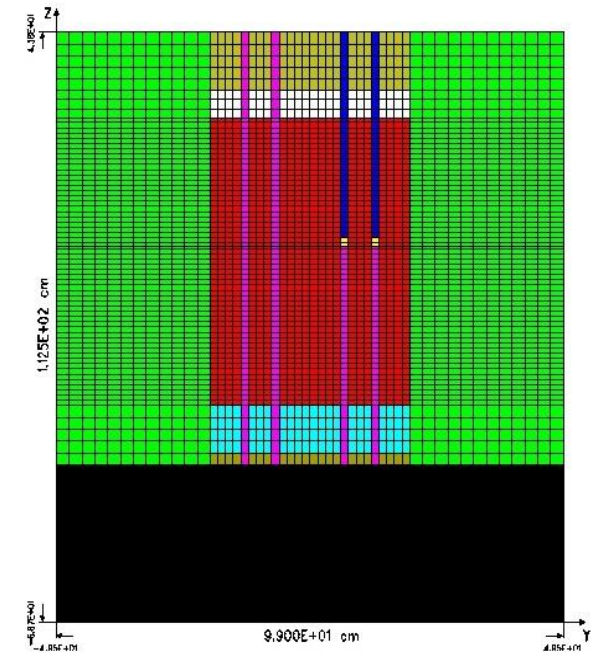

Figure 3. 2D-YZ view of the reactor

Regarding the results, only the axial distribution of the fission rate of certain rods are

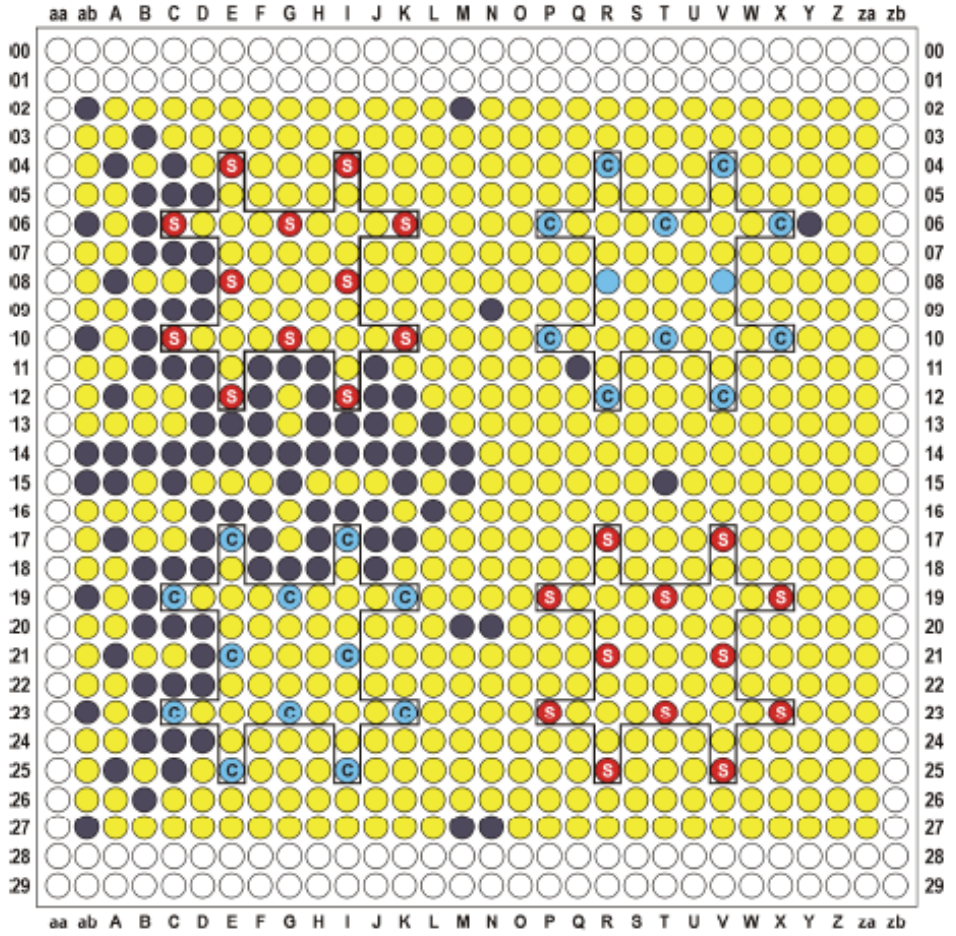

known experimentally. This axial distribution ranges from $2.5 \mathrm{~cm}$ till $52.5 \mathrm{~cm}$. These rods can be seen in the Figure 4 in grey color. The results have been normalized with respect to the value of rod M-14 of this figure, at $22.5 \mathrm{~cm}$ in axial length.
Fuel rod
(C) Control rod
Fuel rod measured with $\mathrm{Nal}$
(5) Safety rod

Figure 4. Core Map Showing the Fuel Rods Measured [6].

With respect to the TORT model adopted, the following approximations have been used: $S_{16}$ for the angular discretization, 16 groups for the energy discretization and spatial discretization of $52 \times 50 \times 82$ meshes. 
Next figures show the rod axial distribution of the relative fission rate for several rods, for a TORT and an MCNP calculation:
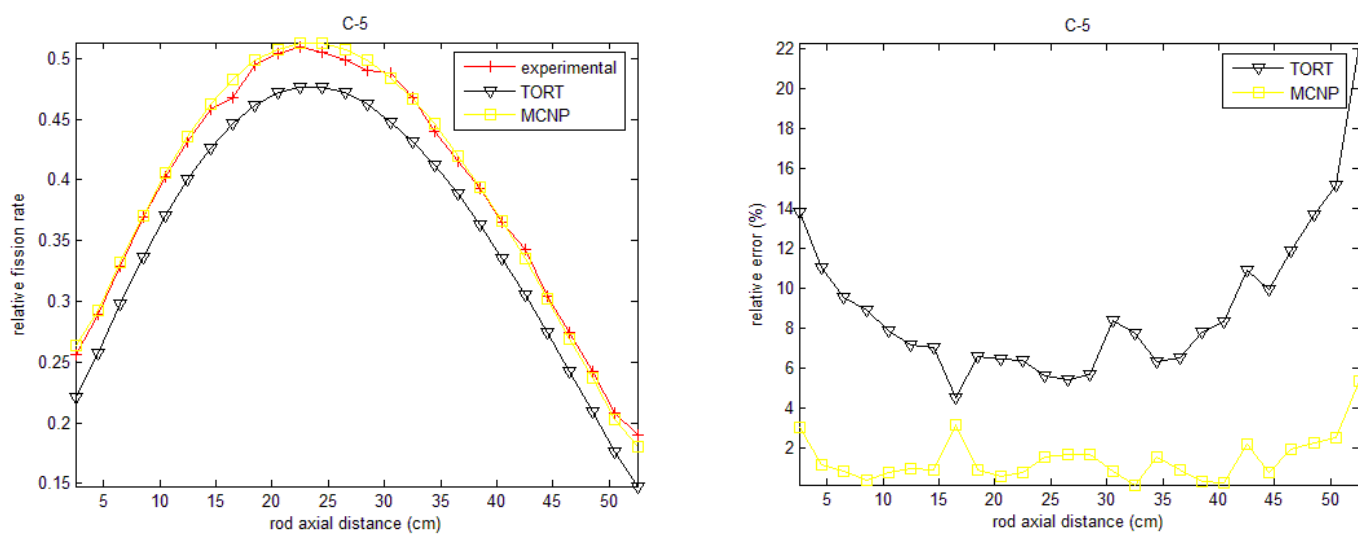

Figure 5. Relative fission rate for C-5 rod. Figure 6. Relative error for C-5 rod.
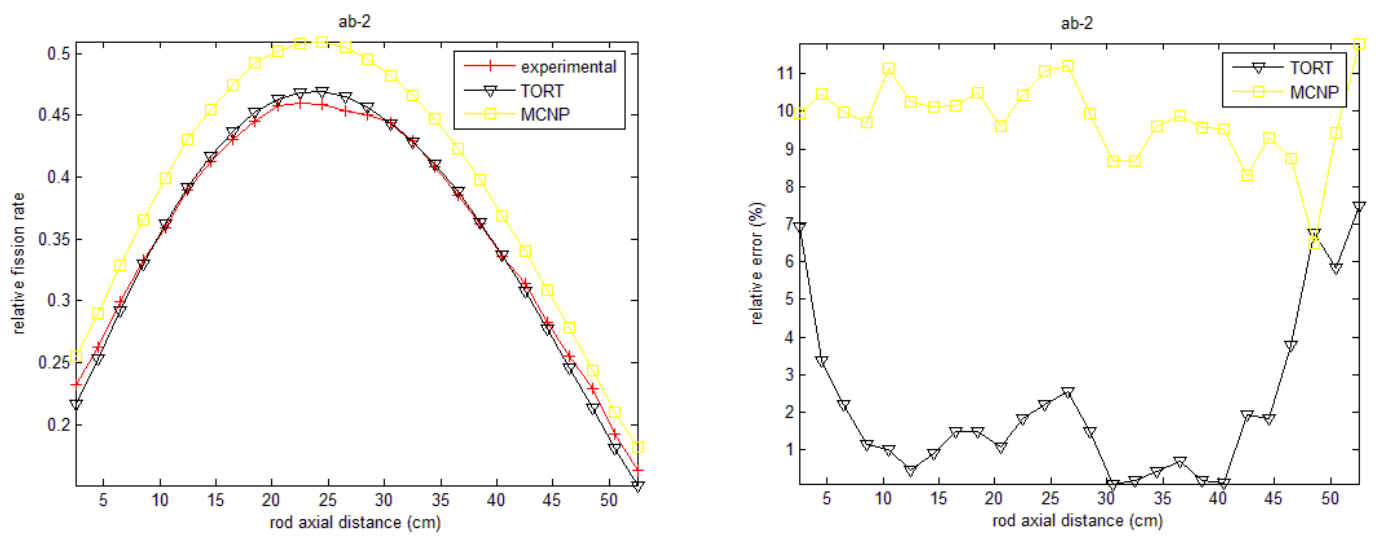

Figure 7. Relative fission rate for ab-2 rod.

Figure 8. Relative error for ab-2 rod.
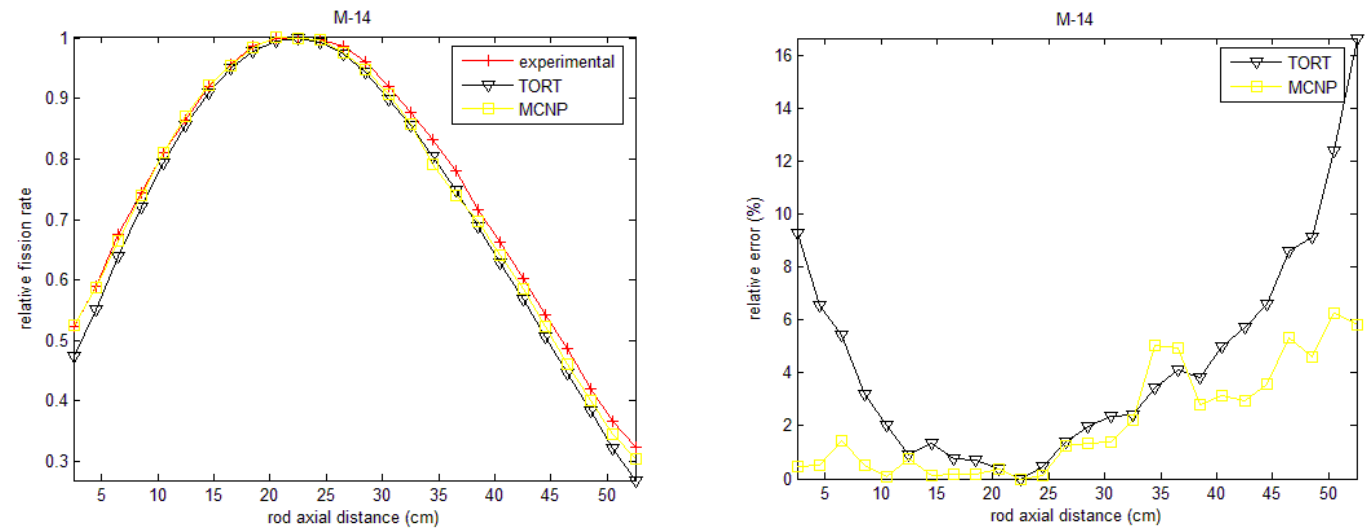

Figure 9. Relative fission rate for M-14 rod. Figure 10. Relative error for M-14 rod. 
The relative error has been calculated as: $\operatorname{Error}(\%)=\frac{|C-E|}{E} \cdot 100$, where $C$ is the value calculated by the code and $E$ the experimental value. The maximum relative error for TORT is $38.37 \%$ and it is located in the rod J-22 of the Figure 4 at $50.5 \mathrm{~cm}$ in axial length. In the case of MCNP, this maximum is $25.08 \%$ and it is located in the same rod but at $52.5 \mathrm{~cm}$. Nevertheless, in some rods the TORT errors range between $0 \%$ and $7 \%$ as in the case of rod ab-2, thus these results are in agreement with the experimental values.

To determine the adequacy of each code, the square error has also been calculated as: Error $^{2}=(C-E)^{2}$. The total sum of the square errors is 1.9708 for MCNP and 3.9169 for TORT. It can be seen the same shape of the distribution for both codes, but MCNP is more accurate than TORT. However, the results are in agreement.

On the other hand, a $K_{\text {eff }}$ of 1.00074 was obtained with TORT, and 1.00500 with MCNP, which are in the same order of magnitude of criticality, but TORT's value is more accurate compared to the expected value of $K_{\text {eff }}=1.00000$.

Moreover, the calculation of TORT spent 759.7 minutes with 1 CPU core, while the calculation of MCNP spent 2934 minutes with 15 cores.

\section{Sensitivity study}

A sensitivity analysis of the TORT quadrature order and mesh has been made. Regarding the quadrature order, $S_{10}$ and $S_{6}$ approximations have been considered, without modifying the reference mesh. In addition, a coarse mesh and a fine mesh were also used, without modifying the reference quadrature order. The coarse one was defined by $23 \times 24 \times 36$ meshes; the fine one by $101 \times 91 \times 57$ meshes.

The next table summarizes the results of the sensitivity analysis: 
Table 1 . Sensitivity study summary.

\begin{tabular}{|c|c|c|c|c|}
\hline \multirow{2}{*}{ Case } & $\boldsymbol{K}_{\text {eff }}$ & $\begin{array}{c}\text { Maximum } \\
\text { relative error } \\
(\%)\end{array}$ & $\begin{array}{c}\text { Total sum of } \\
\text { the square } \\
\text { errors }\end{array}$ & $\begin{array}{c}\text { Calculation } \\
\text { time (minutes) }\end{array}$ \\
\hline $\boldsymbol{S}_{\boldsymbol{1 0}}$ & 1.00132 & 38.42 & 3.9144 & 399 \\
\hline $\boldsymbol{S}_{\boldsymbol{6}}$ & 1.00129 & 38.51 & 3.9383 & 372 \\
\hline Coarse mesh & 0.962034 & 100.88 & 49.0204 & 72 \\
\hline Fine mesh & 0.996796 & 36.71 & 8.4878 & 5888 \\
\hline
\end{tabular}

Moreover, the results of some rods are shown in the next figures:
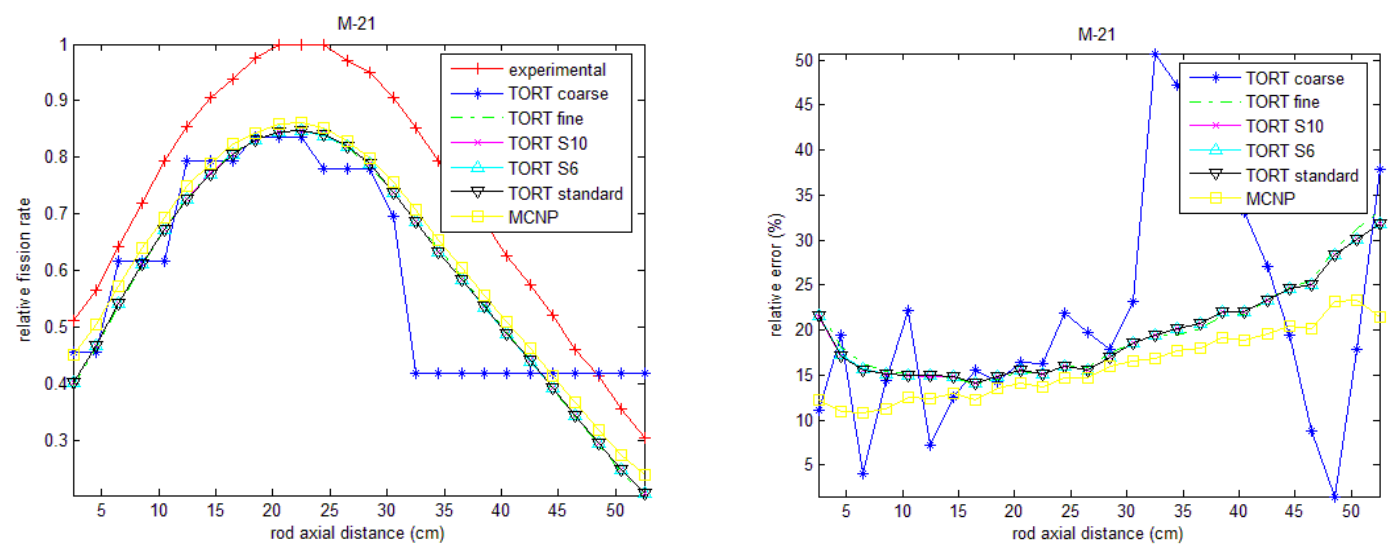

Figure 11. Relative fission rate for M-21 rod. Figure 12. Relative error for M-21 rod.
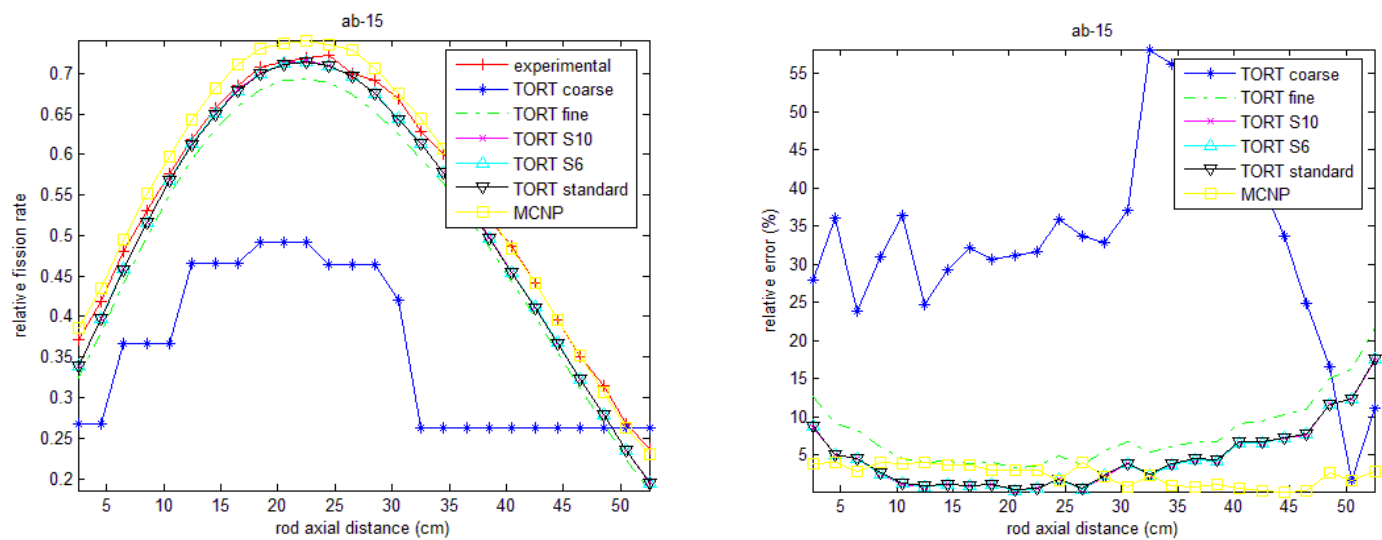

Figure 13. Relative fission rate for ab-15 rod. Figure 14. Relative error for ab-15 rod. 
With respect to the quadrature order, the errors are almost equal to those of the reference case $\left(S_{16}\right)$, this makes sense because of the isotropic media, due to fuel and moderator. However, the calculation time is 2 times slower. Therefore, it is convenient to use less quadrature order because of the fact that it implies a reduction in the calculation time but it does not increase the errors.

Although the coarse mesh spent less time in the calculation, it is unacceptable due to its errors. On the other hand, the fine mesh does not imply a reduction in the error and the calculation time is larger.

\section{Conclusions}

Firstly, there is good agreement between TORT and MCNP calculation, as it can be seen in figures 9 and 10. On the other hand, the computational time of TORT calculation was approximately 16 times less than that of MCNP, so it is an another strength of the method.

However, this paper does not reflect the methodology to generate the multigroup cross sections which can be a difficult task in certain problems. In fact, this methodology is crucial to obtain good results.

Moreover, the computational time can be reduced by using the approximation $S_{6}$, obtaining results as accurate as those obtained with $S_{16}$ approximation. However, the computational time of the quadrature $S_{10}$ is similar to $S_{6}$ and the results are more accurate.

The spatial discretization is crucial to model correctly the reactor and it has a strong influence in the computational time. In this case, the mesh selected is sufficient to solve the problem, and a finer mesh does not improve the results. On the other hand, 
a coarser mesh reduces significantly the calculation time but its results are not accurate enough.

\section{Acknowledgements}

The authors wish to thank Departamento de Engenharia Nuclear da UFMG and Instituto de Pesquisas Energéticas e Nucleares for all data and support.

\section{References}

[1] E. E. Lewis, W. F. Miller, "Computational Methods of Neutron Transport”.

[2] X-5 Monte Carlo Team, "MCNP-A General Monte Carlo N-Particle Transport Code, Version 5 - Vol. I: Overview and Theory", Los Alamos National Laboratory, Report LA-UR-03-1987, 2003.

[3] W. A. Rhoades, R. L. Childs, "DOORS-3.2a, One, Two- and Three-Dimensional Discrete Ordinates Neutron/Photon Transport Code System", RSICC Computer Code Collection CCC-650, Oak Ridge National Laboratory, Oak Ridge, TN.

[4] R. E. MacFarlane, D. W. Muir, "NJOY99.0 Code System for Producing Pointwise and Multigroup Neutron and Photon Cross Sections from ENDF/B Data", PSR480/NJOY99.0, Los Alamos National Laboratory, 2000.

[5] W. A. Rhoades, D. B. Simpson, "The TORT Three-Dimensional Discrete Ordinates Neutron/Photon Transport Code", ORNL/TM-13221, 1997.

[6] International Handbook of Evaluated Reactor Physics Benchmark Experiments, NEA/NSC/DOC(2006)1, 2011. 